\title{
Single-Event Upsets in Microelectronics
}

\section{Henry H.K. Tang and Nils Olsson, Guest Editors}

\begin{abstract}
This article introduces the February 2003 issue of MRS Bulletin on "Single-Event Upsets (SEUs) in Microelectronics." These radiation effects in devices and circuits have been recognized in recent years as a key reliability concern for many current and future silicon-based technologies. This introduction sets the scope for critical discussions on this subject. The articles in the issue reflect the interdisciplinary nature of SEU research. The contributing authors include experts from several specializations: technology reliability, materials science, device physics, circuit designs, and theoretical and experimental nuclear physics. We review the current understanding of SEU problems from the perspectives of radiation physics, circuit design issues, and global technology developments. The discussions cover the key areas of modeling, circuit analyses, accelerator tests and experiments, basic nuclear data, and environmental neutron measurements.
\end{abstract}

Keywords: radiation effects in microelectronics, single-event effects, single-event upsets (SEUs), soft errors.

The scaling of silicon-based integratedcircuit technologies poses a number of challenges for manufacturing. Among these, certain radiation effects known as singleevent upsets (SEUs) have attracted considerable attention in the past decade. ${ }^{1,2}$ They are now recognized in the industry as an important reliability area for many current and future products.

When a charged particle hits a sensitive volume in a microelectronic device, such as a depletion region (a region of unbalanced charge across a $p-n$ junction), the local carriers-electrons and holesinduced by the ionizing particle, under appropriate conditions, can be amplified to form an electrical pulse large enough to flip the memory state of a cell from 1 to 0 or vice versa. This random event, called a single-event upset or soft error, causes data corruption, although it does not cause any permanent damage in the device. Three major sources of SEUs are known to affect commercial products: (1) alpha particles (helium-4 nuclei) emitted by radioactive atoms found in packaging materials, (2) high-energy terrestrial cosmic rays, and (3) thermal neutrons in certain device materials that are heavily doped with ${ }^{10} \mathrm{~B}$. Alpha particles emitted from packaging materials cause SEUs through direct ion- ization processes in the device. A thermal neutron (a low-energy neutron with a kinetic energy in the millielectronvolt range) undergoes a capture reaction with a ${ }^{10} \mathrm{~B}$ nucleus (present, e.g., in certain types of insulating glass placed next to a device), which is followed by the emission of a ${ }^{7} \mathrm{Li}$ nucleus and a ${ }^{4} \mathrm{He}$ nucleus. One of these two nuclei will then hit the device and cause SEUs. The scenario of high-energy cosmic rays is more complex. Subatomic particles like neutrons, protons, and pionscomponents of terrestrial cosmic rays at various altitudes - interact with silicon and the packaging materials of microelectronic devices, producing secondary charged fragments, like alpha particles and heavy recoil nuclei (nuclei remaining after lighter particles have been emitted). These secondary particles ionize the sensitive device regions and cause SEUs. Due to the wide energy range of the cosmic-ray particles and the large variations of their fluxes, shielding that will be effective for all applications is not a practical engineering solution. Hence, it is expected that cosmic rays will continue to play a major role in SEU issues for future technologies, even though solutions may be found to mitigate the problems from alpha particles and thermal neutrons.
This issue of MRS Bulletin has brought together a group of experts from industry, academia, and government laboratories to discuss the current SEU problems. Their articles cover a number of key aspects of this growing field.

The first article, by Tang and Rodbell, gives an overview of the present understanding of SEUs. The authors discuss the three sources of SEUs in terms of their particle origins. The dynamics of a fieldfunneling process-the major chargecollection mechanism responsible for SEUs in bulk complementary metal oxide semiconductor (CMOS) devices-is reviewed. The salient features of nuclear spallation reactions - the principal cause of cosmic ray-induced SEUs - are outlined. Nuclear spallation occurs when a high-energy particle collides with a nucleus, causing it to break up into secondary fragments. The status of SEU research on current and new CMOS devices are summarized.

The second article, by Baumann, discusses SEU issues from a global technology standpoint. Based on product-development trends, the author makes several key observations and projections for the future. Terrestrial cosmic rays are expected to play an important role in SEU reliability. For dynamic random-access memories, SEU sensitivity is relatively unchanged by scaling. However, for static random-access memories, SEU sensitivity is increasing for each new technology. Error detection and correction techniques at the circuit level are the most effective means to improve SEU reliability.

In the third article, Blomgren et al. review some of the current experimental neutron-physics programs in Sweden, which are strongly motivated by SEU research. Discussions focus on the dual mission of quasi-monoenergetic (having energies distributed closely around some peak value) neutron beams: (1) at the basic research level, nuclear data measurements provide crucial input for SEU work; and (2) at the product/engineering level, radiation tests are performed to characterize SEU sensitivity of devices and circuits. The highlights for the next few years include new experiments based on novel inverse kinematics techniques, proposed to measure important recoil cross sections. Inverse kinematics experiments involve firing a heavy atom, like ${ }^{28} \mathrm{Si}$, at a target containing protons or neutrons; this eliminates the target absorption problem encountered when protons or neutrons are fired at a stationary ${ }^{28} \mathrm{Si}$ target.

Currently, some of the best solutions for particle-induced radiation problems in microelectronics are based on circuit tech- 
niques. Hence it is important to examine the circuit aspects of the SEU phenomena. The fourth article, by Bernstein, analyzes SEUs from the perspectives of logic-circuit topologies in high-speed microprocessors, radiation response mechanisms, and circuit techniques to mitigate radiationinduced data corruption.

The article by Goldhagen is a review of state-of-the-art environmental neutron measurements using Bonner spheres (spherical neutron spectrometers) and advanced spectrum unfolding techniques. The measurements cover 12 decades of energy scales (from the thermal energy range up to gigaelectronvolt range). These neutron spectra are crucial input for ab- solute SEU rate calculations, and they are essential for setting new SEU standards for future products.

In addition to SEUs, temporary or permanent material damage on a macroscopic scale occurs when the intensity of radiation increases. This damage generally results in various kinds of gradual or abrupt degradation of device characteristics. The last article, by Foster, reviews the subject of accumulative radiation effects due to displacement damage, which is analyzed in the general framework of non-ionizing energy loss. These radiation effects are particularly important for electronics exposed to harsh radiation environments, such as those used in space programs.
Hence this article extends the intended scope of this issue of MRS Bulletin.

For new products, it is important that SEU reliability issues be properly addressed and that their developments stay on the course set by the International Technology Roadmap for Semiconductors (available at URL http:/ / public.itrs.net). The best strategy for SEUs is to understand their origins and to contain the potential problems at an early stage of the technology cycle.

\section{References}

1. IBM J. Res. Develop. 40 (1) (1996)

2. E. Takeda, E. Murakami, K. Torii, Y. Okuyama, E. Ebe, K. Hinode, and S. Kimura Microelectron. Reliab. 42 (2002) p. 493.

\section{Henry H.K. Tang,}

Guest Editor for this issue of MRS Bulletin, is a member of a materials science group at IBM T.J. Watson Research Center, Yorktown Heights, New York. He received his BA degree (magna cum laude, Phi Beta Kappa, with honors in physics and mathematics) from Kalamazoo College in 1974. He attended Yale University as F.W. Heyl and Elsie L. Heyl Predoctoral Fellow, and was awarded a PhD degree in theoretical physics in 1979. His dissertation focused on two topics: generalized mean-field theory of nuclear many-body systems and hydrodynamic model of heavy ion reactions in the low- and medium-energy regimes. In 1979-1980, Tang was awarded a National Science Foundation Postdoctoral Fellowship in Nuclear Theory from the Massachusetts Institute of Technology (MIT). During this time, he was also a visiting member of the Niels Bohr Institute (NBI) in Copenhagen. In 1980-1982, he returned to MIT, where he was appointed as a Postdoctoral Research Associate at the Center of Theoretical Physics and
Physics Department. In 1983, he joined the research staff of the Cyclotron Institute of the Texas A\&M University (TAMU), where he was also a visiting faculty member in the Physics Department. At NBI, MIT, and TAMU, Tang worked on several topics in nuclear theory, which included transport-theoretic formulation and numerical simulations of heavy ion reactions.

In 1986, Tang joined IBM at East Fishkill, Hopewell Junction, New York, and became a key member of a research group working on single-event upset (SEU) problems in microelectronics. In these efforts, he developed general-purpose nuclear reaction models, and helped to design the industry's first comprehensive system of SEU simulation models and tools. Over the years, Tang mentored several IBM-sponsored university-based experimental nuclear physics programs. He chaired and co-chaired sessions in international conferences on basic and applied nuclear research, and SEU reliability problems. In addition

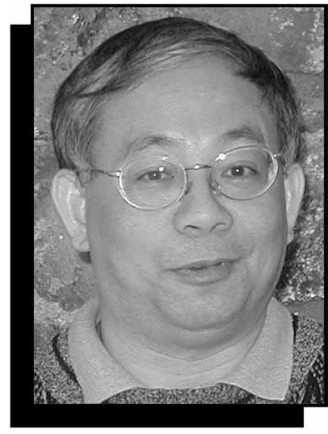

Henry H.K. Tang

to nuclear reactions and radiation physics, Tang worked in the areas of high-field electronic transport in semiconductors and modeling of advanced devices. In 2001, Tang joined IBM's T.J. Watson Research Center to concentrate on new SEU issues and projects. He is a member of the American Physical Society.

Tang can be reached by e-mail at hktang@ us.ibm.com.

Nils Olsson, Guest

Editor for this issue of MRS Bulletin, is research director at the Swedish Defense Research Agency, where he is department head of the Division of Systems

Technology, and Adjunct

Professor of Applied

Nuclear Physics at

Uppsala University,

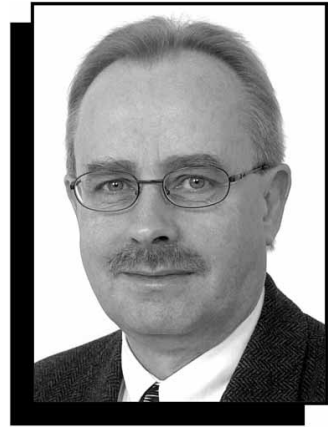

Nils Olsson

where he also received his $\mathrm{PhD}$ degree. His research areas are neutroninduced nuclear reactions and their applications within energy technology, nuclear-waste incineration, cancer therapy, and reliability of electronics. He has over 200 publications in international journals or presentations at international conferences.

Olsson can be reached by e-mail at nils.olsson@ foi.se.

Robert Baumann is a Distinguished Member of the Technical Staff at Texas Instruments (TI), focused on soft errors in advanced static random-access memory and logic devices. Upon joining TI in 1989, he supported the advanced DRAM Development Group in the area of di-

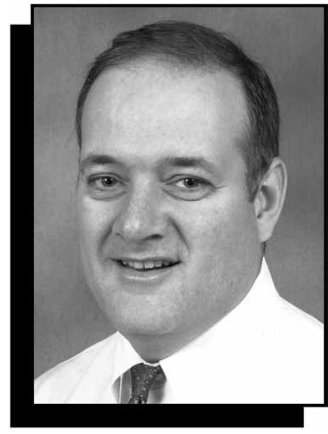

Robert Baumann

electric reliability and radiation-induced soft errors. He pioneered much of TI's soft error program where he made significant contributions to the understanding of alpha and neutron effects including the discovery that activation of ${ }^{10} \mathrm{~B}$ in borophosphosilicate glass by lowenergy neutrons can be a significant source of soft errors in advanced low-voltage technologies. He is one of the primary authors of the JEDEC JESD-89 test specification. Baumann received his BA degree (1984) with honors in physics from Bowdoin College and his PhD degree (1990) in electrical engineering from Rice University, studying process and integration issues for ferroelectrics on silicon devices. 
Baumann is a senior member of the Institute of Electrical and Electronics Engineers (IEEE), chair of the International SEMATECH Committee on Radiation Effects, and co-chair of the Semiconductor Industry Association Technical Steering Committee of Radiation Experts.

Baumann can be reached by e-mail at rbaum@ti.com.

Kerry Bernstein is a senior technical staff member and lead technologist in the High Speed Microprocessor Business Group at IBM Microelectronic Division's Essex Junction, Vermont, facility. He is responsible for future product technology definition, performance, and application. Bernstein received his BS degree in electrical engineering from Washington University in St. Louis and joined IBM in 1978. He holds 36 U.S. patents, and is a co-author of three college textbooks and multiple papers on high-speed complementary metal oxide semiconductors. His interests are in the areas of silicon-on-insulator technology and advanced technology implementation.

Bernstein can be reached by e-mail at kbernste@us.ibm.com.

Jan Blomgren is associate professor of applied nuclear physics at the Department of Neutron Research at Uppsala University, Sweden. He received his $\mathrm{PhD}$ degree (1991) at Uppsala University and has been a research associate at Indiana University and assistant professor at Stockholm University.

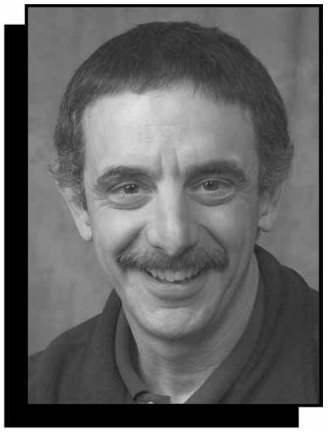

Kerry Bernstein

His research areas are neutron-induced nuclear reactions and their applications within energy technology, nuclearwaste incineration, cancer therapy, and reliability of electronics. He has 90 publications in international journals or presentations at international conferences.

Blomgren can be reached by e-mail at jan.blomgren@tsl.uu.se.

Charles C. Foster retired from the Indiana University Cyclotron Facility (IUCF) in February 2001 and is now coowner of Foster Consulting Services, L.L.C. He received his BS degree with honors in physics from Tulane University in 1957. His honors thesis was in beta-ray spectroscopy. He served as a naval officer after graduation from Tulane and then returned to graduate school and continued to work in beta-ray spectroscopy at Tulane as a National Defense Act Fellow. In 1962, he transferred to Indiana University to complete his graduate studies using the university's cyclotron, receiving an MS degree in physics in 1964 and a PhD degree in experimental nuclear physics in 1967. His dissertation was on the measurement of proton polarization in deuteron-

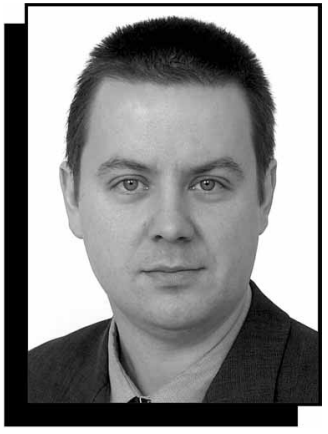

Jan Blomgren

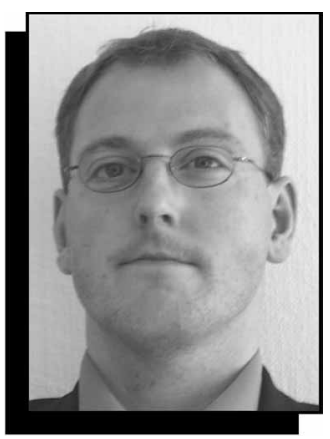

Bo Granbom

induced stripping reactions using a magnetic spectrometer.

Foster was an instructor at Princeton University from 1967 to 1970, where he worked on the design, construction, and commissioning of the Princeton cyclotron, shielding, beamlines, and experimental facilities. From 1970 to 1973, he was an assistant professor of physics at the University of Missouri in St. Louis.

He was the principal investigator on a National Science Foundation grant from 1972 to 1976 for the study of elastic scattering of complex nuclear projectiles at extreme back angles by a magnetic deflection technique, and on a Department of Interior, Office of Water Resources Research Grant from 1972 to 1974 for the development of a portable device for the quantitative detection of trace elements in water in the sub-nanogram sensi-

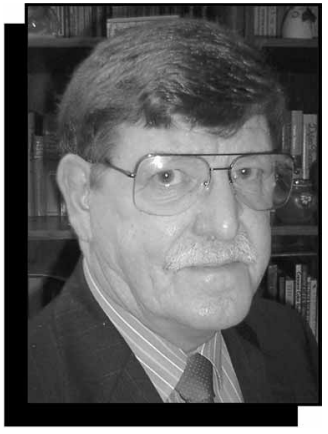

Charles C. Foster

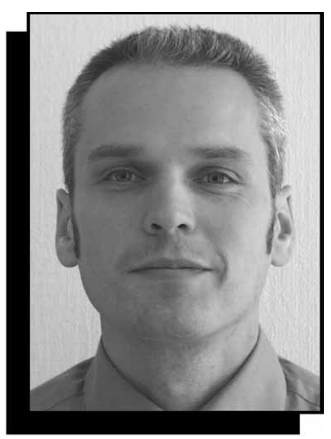

Thomas Granlund

tivity range using alphaparticle-induced $x$-ray fluorescence.

In 1973, Foster returned to Indiana University as a visiting scientist to help with the construction, installation, and commissioning of the beamlines and experimental instrumentation for the university's separated-sector cyclotron then under construction. He served as a staff physicist at IUCF from 1975 to 1980 and as the coordinator of research services from 1980 until retirement. In 1995, he initiated the radiation effects research program at IUCF to make available public access to the high-energy proton beam for study of the radiation response of semiconductor devices and systems to such radiation. He has served as the coordinator of this program from 1995 until late 2002.

Foster is a lifetime member of the American Physical Society and

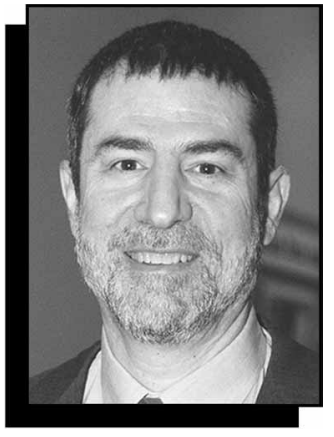

Paul Goldhagen

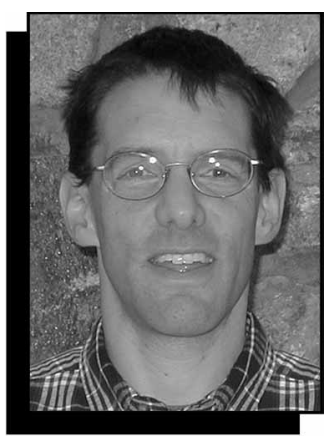

Kenneth P. Rodbell

a member of the Institute of Electrical and Electronics Engineers. He served as chair of the Dosimetry Session of the 1999 Nuclear and Space Radiation Effects Conference. He is author or co-author of over 150 papers in refereed scientific journals.

Foster can be reached by e-mail at fosterchc@ attbi.com.

Paul Goldhagen is a physicist at the Environmental Measurements Laboratory (EML) of the U.S. Department of Energy. (EML is now transitioning to the U.S. Department of Homeland Security). His main research interest is the measurement of neutron fluxes and energy spectra at environmental levels, including neutrons from cosmic radiation aboard aircraft and on the ground, from fission and fusion reactors, and from nuclear materials. He has also done research in the trans- 
port, detection, dosimetry, and microdosimetry of various types of radiation. He received a $\mathrm{BSc}$ degree in physics from the University of Maryland in 1967 and a PhD degree in physics from Princeton University in 1973 . He worked in experimental elementary particle physics and radiological physics at Princeton, the Massachusetts Institute of Technology, Rutgers University, and the College of Physicians and Surgeons of

Columbia University.

Goldhagen can be reached at U.S. Department of Homeland Security, 201 Varick Street, 5th Floor, New York,
New York 10014-4811, USA, and by e-mail at Paul.Goldhagen@ eml.doe.gov.

Bo Granbom is a technology coordinator at Saab Avionics AB Sweden. He has studied fault tolerant systems at Linköping University in Sweden. His present position involves the technical coordination at both domestic and international grounds in the areas of electromagnetic techniques, microelectronics, fault tolerant techniques, and cosmic radiation effects on semiconductor devices. He has three publications in international journals.
Granbom can be reached by e-mail at bo.granbom@ esavionics.se.

Thomas Granlund is a project manager at Saab Avionics AB, Sweden. He received his $\mathrm{PhD}$ degree (2000) at Linköping University in Sweden. His research area then was electricalconducting and electroluminescent polymers. His present research area is cosmic radiation effects on semiconductor devices. His present position involves technical knowledge in the areas of electromagnetic techniques and fault tolerant techniques. He has 10 publi- cations in international

journals.

Granlund can be reached by e-mail at thomas.granlund@ esavionics.se.

Kenneth P. Rodbell is a research staff member at the IBM Thomas J. Watson Research Center in Yorktown Heights, New York. He joined IBM Research in 1989 after spending three years at the IBM Semiconductor Development Laboratory in East Fishkill, New York Rodbell's research interests are in Si-based electronic materials, specifically thin-film metallurgy and crystallographic texture, electromigration, chemical-mechanical polishing, $1 / f$ noise in metallic films, positron annihilation spectroscopy, radiation-induced soft errors, and low- $\kappa$ dielectrics.

He received his BS (1982) and MS (1983) degrees from Rensselaer Polytechnic Institute (RPI), and his PhD degree in materials science and engineering with a minor in statistics (1986), also from RPI. Rodbell has co-authored more than 90 publications; he has 24 U.S. patents and has given numerous invited talks.

Rodbell can be reached by e-mail at rodbell@us.ibm.com.

\section{The Materials Gatecway: www.mrs.org}

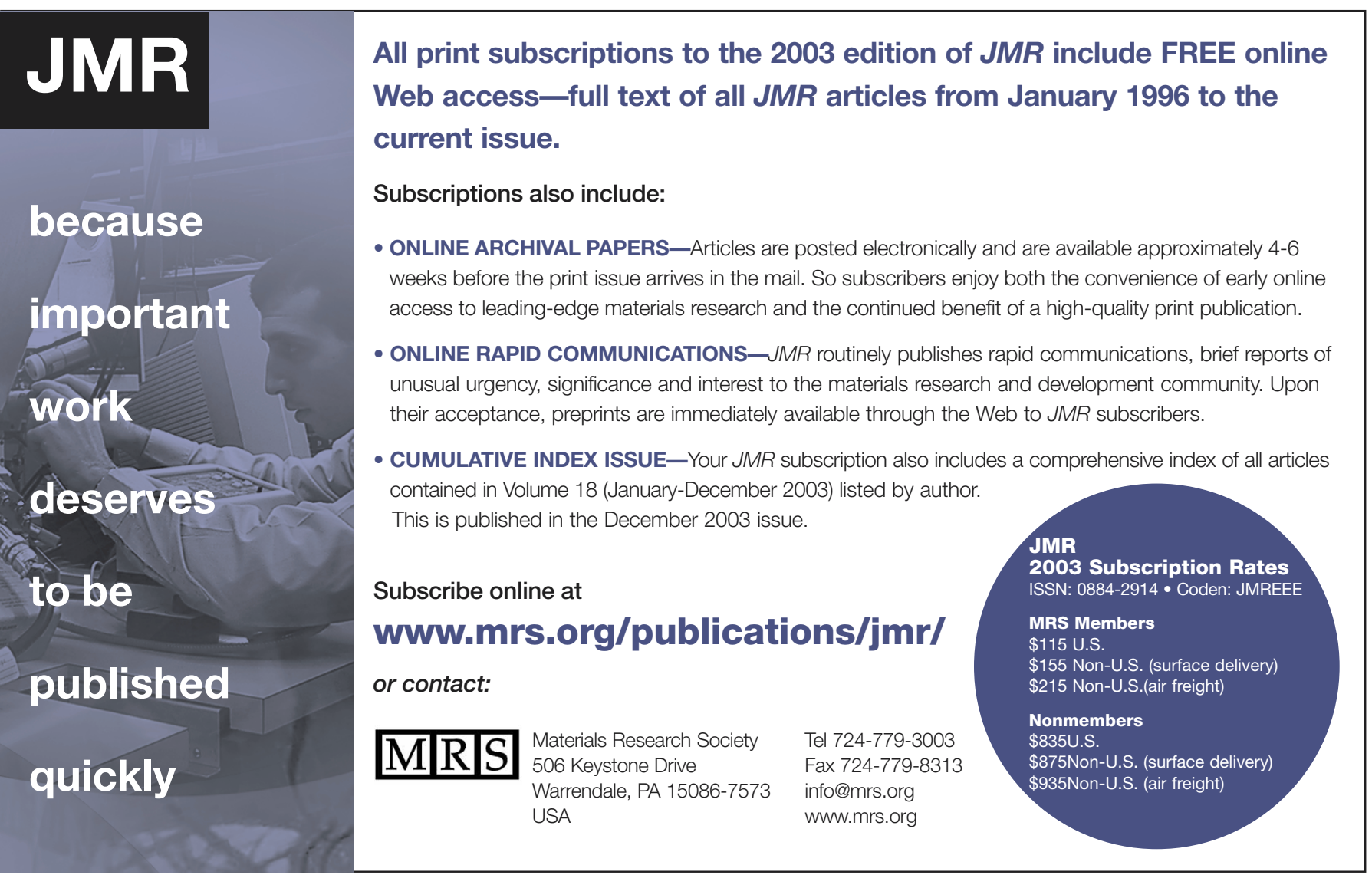

